\title{
Emtricitabine/Rilpivirine/Tenofovir Alafenamide Fumarate
}

National Cancer Institute

\section{Source}

National Cancer Institute. Emtricitabine/Rilpivirine/Tenofovir Alafenamide Fumarate. NCI

Thesaurus. Code C157539.

\begin{abstract}
A fixed combination of emtricitabine, a nucleoside reverse transcriptase (RT) inhibitor (NRTI) analog of cytidine; rilpivirine, a diarylpyrimidine derivative and non-nucleoside RT inhibitor (NNRTI); and tenofovir alafenamide fumarate, the fumarate salt form of the lipophilic phosphonamidate prodrug of tenofovir, a NRTI analog of adenosine monophosphate, that is used to treat human immunodeficiency virus (HIV) infection. Upon oral administration, emtricitabine and tenofovir inhibit the activity of HIV RT by competing with natural deoxyribonucleosides for incorporation into the growing viral DNA chain resulting in early chain termination. This interferes with the generation of DNA copies of viral RNA, which is necessary for the synthesis of new virions. Rilpivirine, a noncompetitive NNRTI binds to RT and inhibits RNA and DNA-dependent polymerase activities, including viral replication.
\end{abstract}

